\title{
Cardiac Arrest in a Patient with Polyarteritis Nodosa
}

\author{
Yuko Harada $^{1}$, Takahiro Suzuki ${ }^{2}$, Toshihito Shinagawa ${ }^{3}$ and Tatsuji Yoshimoto ${ }^{1}$
}

\begin{abstract}
Polyarteritis nodosa (PAN) is a rare necrotizing vasculitis that occurs in small- to medium-sized muscular arteries. A 71-year-old man was admitted to our hospital for an evaluation of a sustained fever and he later died of cardiac arrest. The autopsy revealed that the patient had PAN and severe coronary vasculitis with no signs of cardiac ischemia. The reason for the patient's sudden death remains unclear; however, cardiac arrest due to coronary vasculitis was suggested. This is a rare case of PAN coronary vasculitis that led to sudden death without either stenosis or any embolism.
\end{abstract}

Key words: polyarteritis nodosa, coronary vasculitis, cardiac arrest, sudden death

(Intern Med 52: 2759-2763, 2013)

(DOI: 10.2169/internalmedicine.52.1090)

\section{Introduction}

Polyarteritis nodosa (PAN) is a rare necrotizing vasculitis that occurs in small- to medium-sized muscular arteries. It is known that the coronary arteries are sometimes affected by PAN. We experienced a case of cardiac arrest in a patient diagnosed with PAN postmortem at autopsy. The cause of cardiac arrest remains unclear, even with a histological evaluation.

\section{Case Report}

A 71-year-old man visited our hospital due to a sustained dry cough. A chest X-ray showed infiltration in the right lower lung field and left middle lung field. The white blood cell count was $6,700 / \mu \mathrm{L}$ and the C-reactive protein (CRP) level was $25.60 \mathrm{mg} / \mathrm{dL}$. Serum IgA for Chlamydia pneumoniae was positive; therefore, the patient was suspected to have atypical pneumonia. pazufloxacin (PZFX) (1,000 mg/ day) was administered to treat the pneumonia, and the CRP level decreased to $1.04 \mathrm{mg} / \mathrm{dL}$.

Two months later, the patient developed chest pain and visited the hospital again. An electrocardiogram (ECG) showed ST-depression in the $\mathrm{V}_{5}$ and $\mathrm{V}_{6}$ leads, which led to a diagnosis of unstable angina. The chest pain resolved following treatment with isosorbide mononitrate. The next day, the patient exhibited a high fever of $37.8^{\circ} \mathrm{C}$ and cough. Piperacillin (PIPC) (2 g/day) was administered, and the CRP level decreased from $3.77 \mathrm{mg} / \mathrm{dL}$ to $0.87 \mathrm{mg} / \mathrm{dL}$. The patient refused to undergo cardiac catheterization.

Three months later, he experienced a sustained fever, dry mouth and appetite loss that lasted for two weeks. The white blood cell count and CRP level were remarkably elevated; therefore, he was admitted to the hospital for a further evaluation. His past medical history included asthma.

An examination performed on admission revealed a middle-sized man of $160.8 \mathrm{~cm}$ in height and $72.6 \mathrm{~kg}$ in weight, with the following vital signs: body temperature, $38.1^{\circ} \mathrm{C}$; heart rate, 100 beats/min; blood pressure, $124 / 80$ $\mathrm{mmHg}$. His heart and lung sounds were normal. His abdomen was soft and flat with no tenderness. His extremities were not edematous; however, he reported a tingling sensation in his legs.

The complete blood count $(\mathrm{CBC})$ count revealed an increased level of white cells at $16,700 / \mu \mathrm{L}$ (neutrocytes: 96.0\%, lymphocytes: $2.0 \%$, eosinophils: $1.0 \%$, monocytes: $1.0 \%$ ), a low hemoglobin level of $11.2 \mathrm{~g} / \mathrm{dL}$ and an increased platelet level of $484,000 / \mu \mathrm{L}$. The albumin level was decreased to $2.0 \mathrm{~g} / \mathrm{dL}$, the blood urea nitrogen (BUN) level was increased to $22.4 \mathrm{mg} / \mathrm{dL}$, the alanine aspartate aminotransferase (AST) level was increased to $75 \mathrm{IU} / \mathrm{L}$, the alanine aminotransferase (ALT) level was increased to 61 IU/L, the alkaline phosphatase (ALP) level was increased to

${ }^{1}$ Department of Cardiology, Kawasaki Municipal Ida Hospital, Japan, ${ }^{2}$ Department of Rheumatology, Kawasaki Municipal Ida Hospital, Japan and ${ }^{3}$ Department of Pathology, Kawasaki Municipal Ida Hospital, Japan

Received for publication May 31, 2013; Accepted for publication July 24, 2013

Correspondence to Dr. Yuko Harada, adayuko1219@yahoo.co.jp 


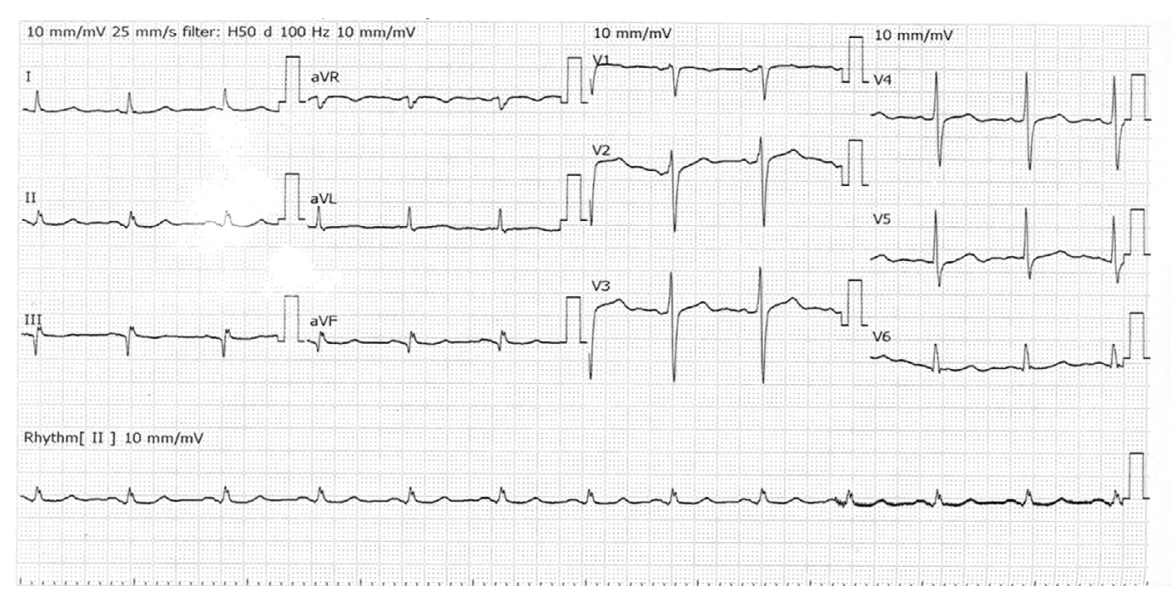

Figure 1. Electrocardiogram obtained on admission.Left from top: I, II, III, aVR, aVL, aVF.Right from top: V1, V2, V3, V4, V5, V6.The electrocardiogram shows a normal sinus rhythm with abnormal Q-waves in the III and aVF leads.

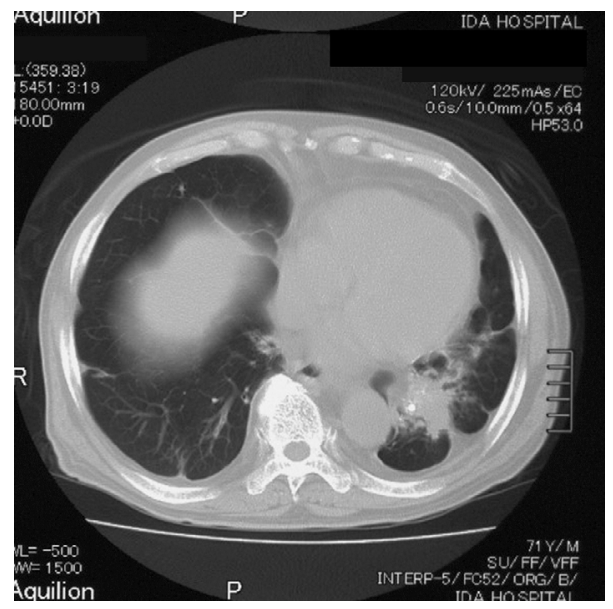

Figure 2. A chest CT scan obtained on admission showing a tumor-like shadow and infiltration in the left lower lobe.

$641 \mathrm{IU} / \mathrm{L}$, the $\gamma$-glutamyl transpeptidase $(\gamma$-GTP) level was increased to $130 \mathrm{IU} / \mathrm{L}$ and the CRP level was increased to $24.53 \mathrm{mg} / \mathrm{dL}$. A urine test showed microscopic hematuria of 10-19/HPF.

An electrocardiogram demonstrated a normal sinus rhythm with abnormal Q-waves in the III and aVF leads (Fig. 1). An echocardiogram showed mild aortic regurgitation with an Ejection Fraction (EF) of $61.4 \%$ and a small amount of pericardial effusion. An abdominal echogram showed mild enlargement of the intrahepatic bile duct, a right renal cyst measuring $5.1 \mathrm{~mm}$ and left renal cysts measuring $76.1 \mathrm{~mm}$ and $12.4 \mathrm{~mm}$. A chest X-ray revealed cardiomegaly and a shadow in the left lower lung field. A chest CT scan disclosed a tumor-like shadow and infiltration in the left lower lobe (Fig. 2).

The findings on the chest X-ray and CT scans were suggestive of pneumonia. However, the high fever and increased CRP level persisted, even with the administration of antibiotics. Initially, meropenem (MEPM, 1 g/day) was administered, followed by ciprofloxacin (CPFX) $(500 \mathrm{mg} /$ day $)$ and Gamma-globulin; however, the WBC count and CRP level remained high (Fig. 3). Sputum, urine, stool and blood cultures were all negative. On the second day of admission, the patient developed pain in his back. On the third day, he developed pain in his legs that persisted until the last day of hospitalization. On the 17th day of admission, he developed sudden severe chest pain early in the morning. An electrocardiogram showed negative T-waves in the V4, V5 and V6 leads (Fig. 4). Subsequently, the patient received nitroglycerin at a dose of $0.3 \mathrm{mg}$ orally. The electrocardiogram monitor demonstrated sinus tachycardia with the same ST-T level. However, only 15 minutes after the patient received the nitroglycerin, he went into cardiac arrest. The electrocardiogram monitor continued to show sinus tachycardia; however, the patient was pulseless. Cardio pulmonary resuscitation was not effective, and he died.

An autopsy revealed fibrinoid necrotizing arteritis in various organs, including the kidneys, brain, heart (coronary arteries), liver, tongue, spleen and pleura. The abnormal findings in the lungs were found to be signs of severe arteritis with scar tissue, which indicates the end stage of fibrinoid necrotizing arteritis (Fig. 5). Therefore, the pathological diagnosis was PAN. However, the cause of the cardiac arrest was unclear. There were no signs of focal ischemia in the myocardium. The coronary arteries did not exhibit any sclerotic changes, narrowing or occlusion. The right coronary artery (RCA), left anterior descending artery (LAD) and their branches showed remarkable infiltration of granulocytes (Fig. 6a, b). Multiple cerebral infarctions were found in the brain, primarily in the basal ganglia, and were excluded from as a cause of the cardiac arrest.

\section{Discussion}

The present patient was diagnosed with PAN after his death. The clinical course was only seven months and it ended in the occurrence of cardiac arrest.

According to the American College of Rheumatology 


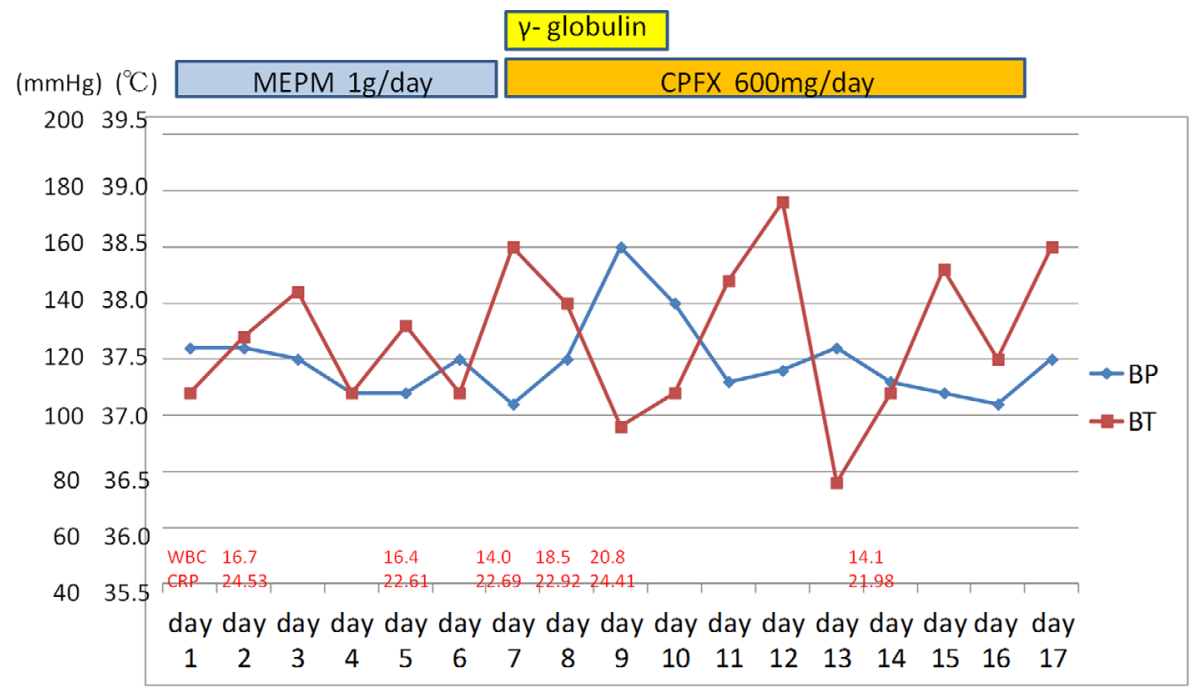

Figure 3. Treatment and clinical course. The patient's blood pressure was not high, except on Day 9. The patient's body temperature, WBC count and CRP level remained high, even after treatment with MEPM, CPFX and Gamma-globulin. BP: blood pressure, BT: body temperature

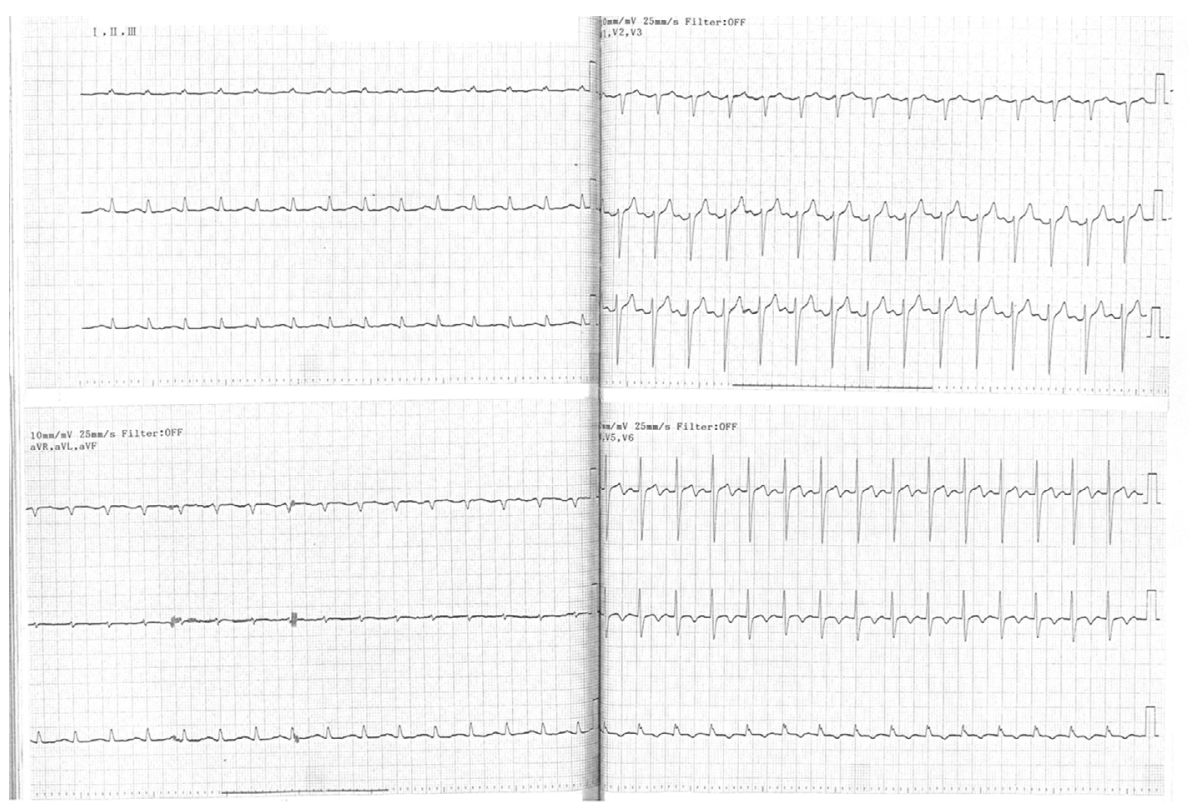

Figure 4. Electrocardiogram obtained during an episode of chest pain. Left from top: I, II, III, aVR, aVL, aVF. Right from top: V1, V2, V3, V4, V5, V6. Negative T-waves are observed in the V4, V5 and V6 leads.

1990 criteria for the classification of PAN, at least three of 10 criteria must be present (1). However, this case met only one criterion: myalgia in the leg muscles. The patient did not report any testicular pain. Dysphagia may be considered a mononeuropathy, which meets another criterion. However, the patient did not experience weight loss, livedo reticularis, a high diastolic blood pressure, elevated levels of BUN or creatinine or positivity for hepatitis B surface antigens. Therefore, it was impossible to make a diagnosis before his death.

The diagnosis was made on a pathological examination performed at autopsy; however, the cause of death remained unclear. Apparently, the patient developed cardiac arrest followed by chest pain. However, the electrocardiogram did not show any ischemic changes immediately before the cardiac arrest, and a pathological examination did not reveal focal ischemia in the cardiac muscle. The coronary arteries exhibited severe arteritis; however, no occlusion or narrowing were found.

There are several reports of PAN occurring in patients with coronary artery disease. Most of these patients had myocardial infarctions due to angiographically or pathologically diagnosed narrowing or occlusion of the coronary arteries. Multiple vascular aneurysms is the most typical angi- 


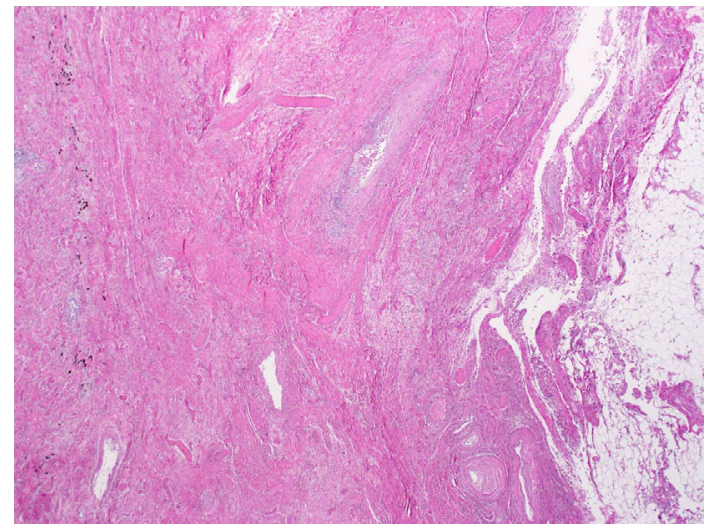

Figure 5. The lungs and pleura.The mass formed on the bottom of the left lung was found to be scar tissue, which indicates the end stage of fibrinoid necrotizing arteritis.

ographic finding of PAN and is also one of the criteria for the disease (1). Some case reports have demonstrated aneurysms and stenotic changes in the coronary arteries (2-4). A few case reports have described acute myocardial infarctions with coronary dissection $(5,6)$. It would be easier to explain the cause of cardiac arrest in our patient if we could prove the presence of stenosis or occlusion in the coronary arteries or ischemic changes in the myocardium.

Rotem et al. concluded that coronary vasculitis is a rare cause of sudden cardiac death after reviewing the previous literature (7). They reported a case of isolated coronary vasculitis with $90 \%$ narrowing of the proximal left anterior descending artery that may have caused sudden death; however, a histological examination did not demonstrate any extensive myocardial infarctions. Christopher et al. reported three cases of sudden death due to coronary vasculitis, all of which involved marked narrowing and acute thrombotic occlusion of the coronary arteries (8). Therefore, coronary arteritis induced by PAN may cause sudden death without atherosclerotic changes or aneurysms, although coronary artery stenosis or occlusion is usually observed.

There is one case report of an acute myocardial infarction with normal coronary arteries in a patient with PAN (9). The authors suggested that the cause of infarction may have been a coronary artery spasm, referring to the presence of vasospastic abnormalities in the peripheral circulation in PAN patients, including a form of Raynaud's phenomenon with an increased propensity for coronary spasms (10). We suggest that our patient may have had a coronary artery spasm that led to cardiac arrest.

Another speculation is that the sinus node or other impulse conduction systems of the heart may have been affected by PAN. A histological analysis revealed that inflammation was extremely severe in the branches of the right coronary artery. It is unclear how coronary arteritis affects the impulse conduction system, and it is impossible to investigate this phenomenon after death.

PAN is treatable with immunosuppressants, including steroids; however, it is sometimes difficult to make an accurate

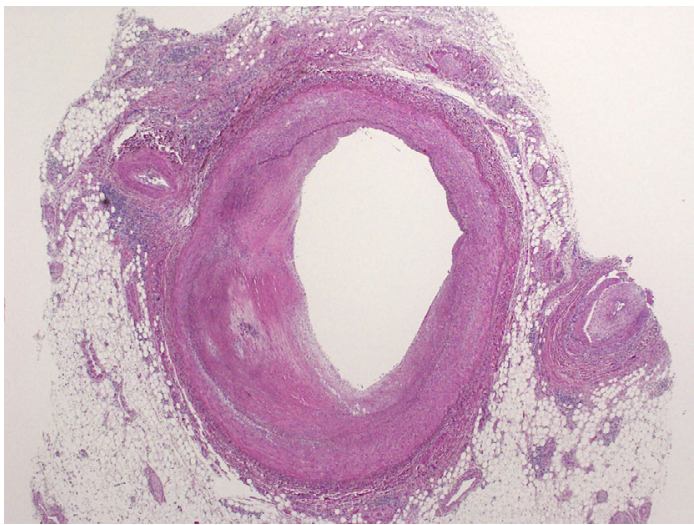

a

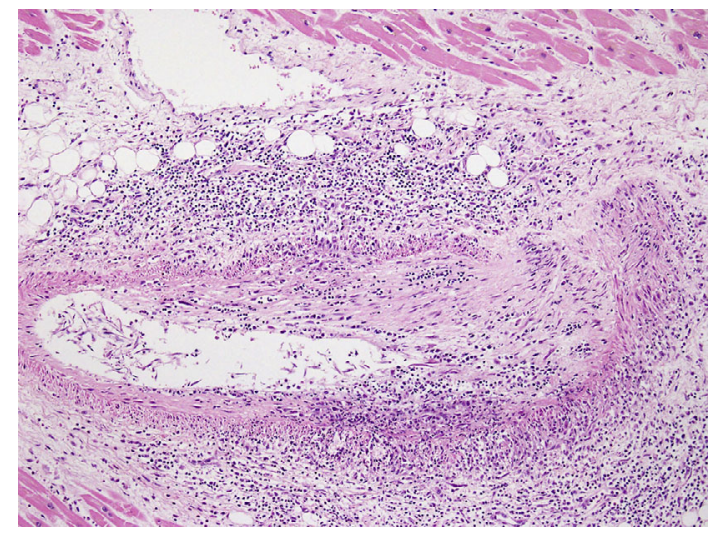

b

Figure 6. a: The RCA and its branches (low-power field). The coronary arteries exhibited remarkable infiltration of granulocytes. b: The branch of the RCA (high-power field). The infiltration of granulocytes on the outer membrane is remarkable.

diagnosis. Untreated PAN may lead to sudden death, as seen in the present case. The cause of cardiac arrest in patients with PAN is not always stenosis or occlusion of the coronary artery. Coronary artery spasms or the destruction of the heart conduction system is also thought to cause cardiac arrest in patients with severe coronary vasculitis induced by PAN.

The authors state that they have no Conflict of Interest (COI).

\section{References}

1. Lightfoot RW, Michel BA, Bloch DA, et al. The American College of Rheumatology 1990 criteria for the classification of polyarteritis nodosa. Arthritis Rheum 33: 1088-1093, 1990.

2. Jin W, Hyun HC, Chan JL, et al. Acute myocardial infarction due to polyarteritis nodosa in a young female patient. Korean Circ J 40: 197-200, 2010.

3. Kastner D, Gaffney M, Tak T. Polyarteritis nodosa and myocardial infarction. Can J Cardiol 16: 515-518, 2000.

4. Przybojewski JZ. Polyarteritis nodosa in the adult. Report of a case with repeated myocardial infarction and a review of cardiac involvement. S Afr Med J 60: 512-518, 1981.

5. Chu KH, Menapace FJ, Blankenship JC, Hausch R, Harrington T. 
Polyarteritis nodosa presenting as acute myocardial infarction with coronary dissection. Cathet Cardiovasc Diagn 44: 320-324, 1998.

6. Canpolat C, Dural M, Atalar E. Acute inferior myocardial infarction in a young female patient with polyarteritis nodosa. Herz 37: 461-463, 2012.

7. Rotem K, Alon E, Zamir D, Rutti H, Sylvia L, Howard A. Isolated coronary vasculitis as a cause of unexpected sudden death. IMAJ 11: 769-770, 2009.

8. Christopher IS, Sara KR, Valarie JR. Sudden death due to unsus- pected coronary vasculitis. Am J Forensic Med Pathol 12: 306312, 1991.

9. Rajani RM, Dalvi BV, D'Silva SA, Lokhandwala YY, Kale PA. Acute myocardial infarction with normal coronary arteries in a case of polyarteritis nodosa: possible role of coronary artery spasm. Postgrad Med J 67: 78-80, 1991.

10. Miklozek CL, Crumpacker CS, Royal HD, Come PC, Sullivan JL, Abelman WH. Myocarditis presenting as acute myocardial infarction. Am Heart J 115: 768-776, 1987.

(C) 2013 The Japanese Society of Internal Medicine http://www.naika.or.jp/imonline/index.html 